\title{
Category Learning in a Transitive Inference Paradigm
}

\author{
Greg Jensen ${ }^{1,5}$, Tina Kao ${ }^{2,3}$, Charlotte Michaelcheck ${ }^{2}$, Saani Simms Borge ${ }^{2}$, Vincent P. \\ Ferrera $^{1,4,5}$, and Herbert S. Terrace ${ }^{2,4}$
}

\author{
${ }^{1}$ Dept. of Neuroscience, Columbia University Medical Center \\ ${ }^{2}$ Dept. of Psychology, Columbia University \\ ${ }^{3}$ Dept. of Psychology, New York City College of Technology, CUNY \\ ${ }^{4}$ Dept. of Psychiatry, Columbia University Medical Center \\ ${ }^{5}$ Zuckerman Mind Brain Behavior Institute, Columbia University
}

\section{ABSTRACT}

The implied order of a ranked set of visual images can be learned by transitive inference, without reliance on stimulus features that explicitly signal their order. Such learning is difficult to explain by associative mechanisms but can be accounted for by cognitive representations and processes such as transitive inference. Our study seeks to determine if those representations may be applied to categories of images without explicit verbal instruction. Specifically, we asked whether participants can (a) infer that images being presented belonged to familiar categories, even when every image presented during every trial is unique, and (b) perform transitive inferences about the ordering of those categories. To address these questions, we compared the performance of humans during a standard TI task, which used the same set of images throughout the session, to performance in a category TI tasks, which drew images from a set of categories. Each of the images used in the category TI task was only presented once, limiting the extent to which stimulus-outcome associations could be learned. Participants were able to learn the order of the categories based on transitive inference. However, participants in the category TI condition did not produce a symbolic distance effect. These findings collectively suggest that differing cognitive processes may underpin serial learning when learning about specific stimuli versus stimulus categories.

Keywords: transitive inference, categorization, serial learning

Transitive inference (TI) is the ability to infer that $\mathrm{A}>\mathrm{C}$, given the premise pairs $\mathrm{A}>\mathrm{B}$ and $\mathrm{B}>$ C. Although initially thought to reflect the capacity for formal logic and appear relatively late in child development (e.g. Piaget, 1921), TI has turned out to be much more general. Rather than using word problems, Bryant and Trabasso (1971)demonstrated that TI could be observed in younger children by letting them make repeated choices among pairs of stimuli and letting them learn the order by trial and error. This approach has since been adapted to many other species, including monkeys (McGonigle and Chalmers, 1977, 1992), mice (Silverman et al., 2015; Van der Jeugd et al., 2009), rats (David, 1992), pigeons (Lazareva and Wasserman, 2006), crows (Lazareva et al., 2004), and even fish (Grosenick et al., 2007) and wasps (Tibbetts et al., 2019). This broad comparative foundation appears to support the claim that TI is a well-preserved cognitive ability with deep evolutionary roots (Vasconcelos, 2008; Jensen, 2017).

The definitive test of TI is to train subjects on only the adjacent pairs in a list, then test performance on novel pairs that are not adjacent and don't include terminal items. For example, to train a five-item list, $\mathrm{ABCDE}$, training would consist of presentation of the four adjacent pairs (AB, BC, $\mathrm{CD}$, and $\mathrm{DE}$ ). At test, the pair BD is considered a "critical pair" because B and D both have equal expected rates of 
reward during training, ruling out associative mechanisms. Exceeding chance levels of accuracy for the first presentation of BD is interpreted as evidence that a transitive inference has occurred.

Despite over a century of study and its demonstration in dozens of species, the mechanisms underlying TI remain unclear. In order to probe this process, studies have not only examined whether individuals display TI, but the patterns of error they display. One of the most robust clues about TI is the symbolic distance effect (SDE), a widely observed signature of serial learning seen in both humans (Acuna et al., 2002) and non-humans (D'Amato and Colombo, 1990). An organism is said to display the SDE if choosing between a pair of stimuli is easier when the items are more widely spaced within an ordered list. This may mean an increase in accuracy for widely spaced pairs, a decrease in reaction time, or both. If subjects are trained using lists of length 6 or more, multiple critical pairs of varying symbolic distance can be tested. While any one of these pairs exceeding chance is proof of TI, an SDE is commonly observed among the critical pairs (Jensen, 2017). For example, given the 7-item list ABCDEFG, lower accuracy and slower reaction times would be expected from the critical pairs $\mathrm{BD}$ and $\mathrm{CE}$ than would be expected from the more widely spaced critical pair BF. The presence or absence of the SDE provides a powerful clue as to the structure of the cognitive representation on which TI depends.

Although TI has been exhaustively researched using a fixed set of images as stimuli, much less is known about how TI interacts with broader categories of stimuli. If someone is asked to consider whether "elephants are bigger than rhinos," they are unlikely to call to mind a specific elephant or a specific rhino; instead, the comparison is done at the category level. Since humans are readily able to perform propositional logic using categories, a natural question is to ask whether the trial-and-error TI paradigms used in non-human studies also provide evidence for categorical TI. Tanner et al. (2015) reported that rhesus macaques were able to perform TI on an ordered list of categories (inferring, for example, that Cats $>$ Birds given arbitrary photos of cats and birds). Furthermore, they were able to do so with categories that had not been trained previous to the TI task; in effect, knowledge of the categories was acquired in parallel with subjects inferring their ordering.

Both serial learning (Terrace, 2011) and categorization (Croft and Cruse, 2004) have been identified as potential cognitive prerequisites for language. Additionally, categorization is closely linked to working memory capacity (Lewandowsky, 2011). It has been shown that categorization involves decisions about how to prioritize sensory information according to its behavioral relevance. Faces, for example, are a socially relevant stimulus category that has an identified neural substrate in humans, monkeys, dogs, and sheep (Kanwisher et al., 1997; Tsao et al., 2003; Cuaya et al., 2016; Kendrick et al., 2001). However, non-mammalian subjects with radically different neural architectures are nevertheless able to distinguish and categorize human faces (Bogale et al., 2011), an aptitude that is consistent with an inborn capacity for flexible learning, and not merely the exploitation of a conserved circuit with a narrow, specific purpose.

Flexibility is a hallmark of categorization processes (Freedman and Assad, 2016). Humans asked to consider ad hoc categories (such as "a thing that might fall on your head") not only readily identify category members, but also display broad agreement about the semantic structure of the category overall (Neuringer and Jensen, 2010). Even in non-human animals, subjects trained on distinct categories reliably categorize ambiguous stimuli, even if they contain mixtures of features never encountered during training (rhesus macaques, Freedman et al., 2001, 2003) (chimpanzees, Sonnweber et al., 2015), and even doing so in non-visual paradigms such as auditory categorization (budgerigars and zebra finches, Spierings and ten Cate, 2016) and olfactory categorization (dogs, Wright et al., 2017). The boundaries that define categories can shift gradually over time and even from moment to moment (Ferrera et al., 2009; Cromer et al., 2010). This flexibility may help to explain how category learning and serial learning can unfold in parallel during a TI task. 


\section{METHODS}

\section{Participants}

Participants were 110 college undergraduates (76F, 34M) who earned course credit. The experiment was approved by Columbia University's Institutional Review Board (protocol AAAA7861), conforming to the guidelines for human research set forth by the American Psychological Association.

\section{Apparatus}

Participants performed the experiment on a desktop computer (a factory-standard iMac MB953LL/A with $8 \mathrm{~Gb}$ of RAM). Responses were made via a mouse-and-cursor interface. The task was programmed in Javascript (making use of the Node.js and Express modules) and run in the Google Chrome web browser, set to full-screen mode. The relevant task code is included in the electronic supplement.

\section{Procedure}

Prior to the experimental session, all participants were given the following verbal instructions: "During this task, you will see two images presented on the computer screen. Use the mouse to click on the one image you think is the correct image. After you click on the one image, both images will disappear, and either a green check mark will appear on the screen, meaning it was correct, or a red cross will appear on the screen, meaning it was incorrect. Then another trial with two images will occur. Try to make as many correct responses as possible." No time limit was implemented for the experimental session. Further information about the task was withheld until debriefing, which occurred at the end of the experimental session. Participants were randomly assigned to one of two groups: "Category TI" (50 participants) and "Standard TI" (60 participants).

\section{Stimulus Categories}

Stimuli in this experiment were images drawn from seven different categories: birds ( 3,111 images), cats (2,768 images), flowers (3,033 images), hooved mammals (2,535 images), minerals (1,501 images), natural landscapes (1,502 images), and architecture (1,503 images). Examples of these stimuli are presented in Figure 1A. These stimulus sets were deliberately constructed to have a wide variety of colors, compositions, and species in order to minimize reliance on simple stimulus features.

\section{Standard TI}

Participants were presented with pairs of items drawn from 7-item lists, whose order is hereafter denoted as ABCDEFG, where A is the first item, B is the second item, and so forth. As such, "A" does not refer to any particular item or category, but rather, the first position of a list order. For each pair of items presented, the item with the earlier rank was always correct. For example, given the pair AB, selecting A would be considered a correct response, whereas selecting $\mathrm{B}$ would be considered incorrect. However, for the pairs BC, selecting B would be considered correct because of its earlier list position than C.

Each participant in the Standard TI condition learned lists that were constructed from images drawn from each seven different stimulus categories. Each of the image presented were re-presented throughout the experimental session. For example, if a participant was assigned an image of a particular bird as stimulus A, they saw that bird every time stimulus A was presented. Each participant was assigned a different set of stimuli from other participants (so no two participants saw the same bird). Furthermore, the order of the stimuli was counterbalanced across participants, with each stimulus appearing approximately equally often at each list rank. Figure 1B presents an example of a 7-item ordered list. However, it is important to note that participants never saw all seven categories at the same time. They only ever saw stimulus pairs, as described below. 
A

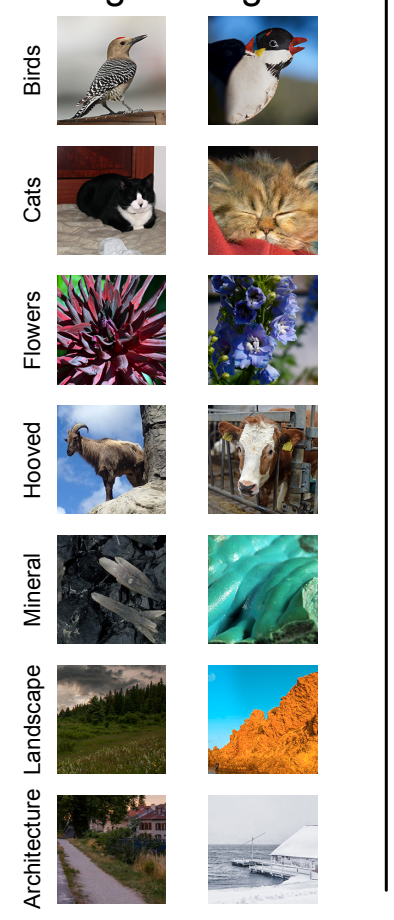

B
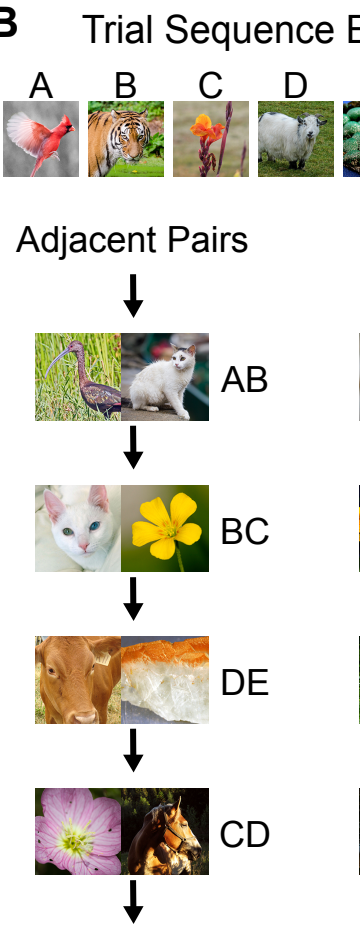
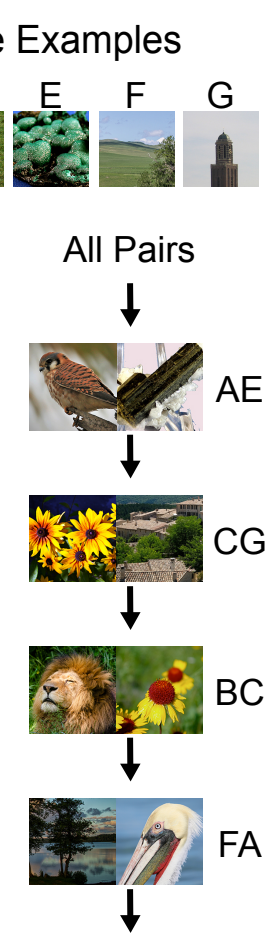

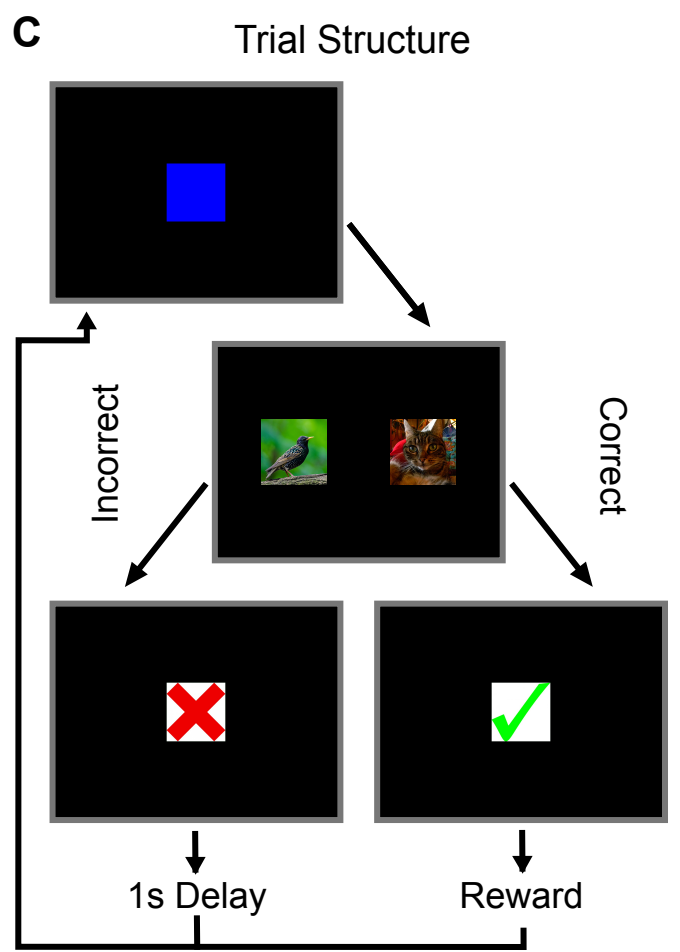

Figure 1. Procedure for the categorical TI task. A. Two exemplars each from the seven stimulus categories used in the experiment. In all categories, an effort was made to include category members from multiple distances and angles, with a mixture of both solitary and group photos, as well as both color and black-and-white. This stimulus diversity was intended to reduce subjects? reliance on specific discrete features as category cues. B. Each phase of the experiment made use of a consistent category sequence (in this case, birds-cats-flowers-hooved-mineral-landscape-architecture). The stimuli themselves, however, were drawn at random from the image bank during every trial. During adjacent-pair trial (using only $\mathrm{AB}, \mathrm{BC}, \mathrm{CD}$, $\mathrm{DE}, \mathrm{EF}$, and FG), the identity of the stimulus changed for every trial, even when the same category appeared in two consecutive trials. The left-right position of stimuli was also counterbalanced. This was also the case during all-pairs sessions, which intermixed all possible stimulus pairings. C. Structure for any single trial of the task. Participants used a mouse to click on a blue square to begin the trial, which is immediately replaced by two images. If a correct response was made, participants saw a green check mark and are immediately given a fluid reward. If an incorrect response was made, subjects saw a red X, followed by a black screen for 1 seconds. Following feedback, the next trial began with the start stimulus.

Each session consisted of a training phase (lasting 588 trials) and a testing phase (lasting 210 trials). During the training phase, only the adjacent pairs were presented, whereas during testing, all pairs were presented. Feedback was given through the experiment, which allowed participants to continue to learn the list order during both the training phase and the testing phase.

Both phases were structured such that each configuration of a pair of items was presented once, before that configuration was presented a second time. For example, during adjacent-pair-only training, a "block" of trials would consist of two presentations each of $\mathrm{AB}, \mathrm{BC}, \mathrm{CD}, \mathrm{DE}, \mathrm{EF}$, and FG, once with the correct item on the left, and once with it on the right, all in a randomized order. All-pairs testing would present each of the 21 pairs twice in a random order, also counterbalancing for screen position. Randomization and counterbalancing were important to prevent participants from exploiting spatial and temporal cues. Thus, training consisted of 49 blocks of 12 trials each, while testing consisted of 5 blocks of 42 trials each. This design ensured that pairs of items were presented in a uniform fashion throughout each session, such that the nth presentation of each stimulus arrangement appeared before the $(n+1)$ th presentation. Feedback at the end of each trial was the only information that could be used to infer list order. 
During a trial, participants were presented with a pair of stimuli, and had to select one stimulus with the mouse to proceed to the next trial. There was no time limit. Selecting the item with an earlier list position was always the correct option, while selecting the other item was always incorrect. Thus, given the pair $\mathrm{BC}$, selecting B would be correct and selecting $\mathrm{C}$ would be incorrect. This trial structure is demonstrated in Figure 1C.

Since the list order was arbitrary, participants had to infer the list order by trial and error. Throughout the experiment, trials advanced at a consistent rate, and aside from the presentation of stimuli, no further cues (e.g. an extra delay) signaled when participants transitioned from training to testing. No verbal instructions aside from those already described above were given. Participants did not interact with the experimenter again until the end of the experiment.

\section{Category TI}

Category TI followed the same protocol as Standard TI, including each participant being assigned a different ordering across the seven categories. So, as in Standard TI, "birds" might correspond to stimulus A for one participant but to stimulus D for another. Trial structure, session length, and other details were also identical in both conditions. Category TI was distinct from Standard TI in only one way: Rather than using the same stimulus as a category exemplar for the whole experiment, participants were instead presented with a random image drawn from the appropriate image bank. For example, although a participant might have "birds" assigned to list position A, each presentation of stimulus A consisted of a different, randomly selected bird photo. Since the instructions did not mention either categories or ordering, participants had to infer from experience what the stimulus categories were, and that they belonged in an order.

\section{Analysis}

To characterize performance during training, we fit a multi-level logistic regression model. Parameters were estimated using the Stan language (Carpenter et al., 2017). The details of our analysis, including the script that implemented the model, are provided in the electronic supplement. This approach allowed us to fit models describing population parameters and to obtain estimates and corresponding uncertainties for individual participant parameters.

During training, the probability of a correct response for each participant was modeled by the logistic function:

$$
p(\text { Correct })=\left(1+\exp \left(-\left(\beta_{\emptyset}+\beta_{t} \cdot t\right)\right)\right)^{-1}
$$

Here, $\beta_{\emptyset}$ is an intercept that represents performance at "trial zero" $(t=0)$ whereas $\beta_{t}$ is a slope term that represents the learning rate over successive trials, t. Participants' parameter values of $\beta_{\emptyset}$ and $\beta_{t}$ were presumed to be drawn from a population distribution that was simultaneously estimated. To allow for the possibility that performance might reach a ceiling lower than $100 \%$ accuracy, the multi-level model's parameters were estimated separately for the first and the last 120 trials of training.

The symbolic distance effect (SDE) of serial learning, observed during all-pairs testing, is the observation that response accuracy increases as a function of the symbolic distance (i.e. difference in rank) between items. Because symbolic distance was potentially a covariate of interest, a more complicated logistic model was used, following the logic of Jensen et al. (2013) and fit using the Stan language:

$$
p(\text { Correct })=\left(1+\exp \left(-\left(\beta_{\emptyset}+\beta_{t} \cdot t+\beta_{D} \cdot D+\beta_{D t} \cdot D \cdot t\right)\right)\right)^{-1}
$$


Here, D denotes the "centered" symbolic distance between stimulus pairs. Since the smallest symbolic distance is 1 , and the largest is 6 , values of D had a value of $\frac{8}{3}$ subtracted to center observations with respect to the mean symbolic distance across all 21 pairs. This facilitated a sampling of the posterior probability distribution and ensured that estimates of both $\beta_{D}$ (the contribution of symbolic distance to the intercept) and $\beta_{D t}$ (the contribution of the symbolic distance to the slope) were uncorrelated with the parameter estimates for the overall slope and intercept. Equation 2 was used to model the test data, since the training phase had no variation in symbolic distance.

It has widely been reported that stimulus pairs that include the terminal items (e.g. AC, or EG) show elevated response accuracy. These so-called "terminal item effects" are potentially explained by the differential rates of reward for choice to A (which is always rewarded) and $G$ (which is never rewarded) (Jensen, 2017).

When using Equation 2 to model performance during the testing phase, we only used those trials that were "critical testing pairs." These pairs consisted of items or categories that were non-adjacent, and non-terminal. For our 7-item list, there were six such critical pairs. Three of these pairs (BD, CE, and $\mathrm{DF}$ ) have a symbolic distance of 2, two (BE and $\mathrm{CF}$ ) have a symbolic distance of 3, while one pair (BF) has a symbolic distance of 4 . This variation in $\mathrm{D}$ is sufficient to get estimates of $\beta_{D}$ and $\beta_{D t}$ without the terminal item effect confounding the result.

We also used Equation 1 to model performance for each of the 21 pairs separately from one another. This provided a picture of learning during testing that was not constrained by any particular model with respect to either symbolic distance effects or terminal item effects.

\section{RESULTS}

Figure 2A (left) shows learning during the first 120 trials of training. Category TI is depicted in black; Standard TI, in blue. In both cases, participants learned the task but, on average, did not do so at the same overall rate. As can be seen in Figure 2C, the posterior distribution for the slope during this early stage of learning overlapped with zero for 60\% (30/50) of Category TI participants, as compared to only $13 \%$ (8/60) of Standard TI participants. This suggests that most Category TI participants were struggling to meet the task demands early in training. By the last 120 trials of training, most participants were no longer showing appreciable learning, having stabilized at a steady state of response accuracy. As shown in Figure 2A (right), mean response accuracy was lower for Category TI participants than for the Standard TI participants. Almost half of the Category TI participants performed at chance (42\%, or 21/50, with intercept estimates overlapping with or below zero); whereas nearly all Standard TI participants performed at high levels of accuracy (only $10 \%$, or $6 / 60$, with intercepts overlapping with zero) during the training phase (Figure 2D).

To assess performance during the testing phase, Equation 2 was used to fit only the six critical pairs (BD, BE, BF, CE, CF, and DF). Estimates of performance on these six pairs, sorted by distance, is presented in Figure 3A. Figure 3 also presents the parameters for the critical-pairs-only model. Although participants on average performed above chance at test (as measured by the model intercepts, Fig. 3B), a substantial number of participants had estimated parameter distributions that overlapped with or were below zero at test $(68 \%$, or $34 / 50$, of Category TI participants vs. $57 \%$, or 34/60, of Standard TI participants). In other words, similar numbers of participants in both groups (between $50 \%$ and $70 \%$ ), displayed performance that could not, on an individual basis, be judged to exceed chance at the start of testing.

A clear difference was evident in the trial slopes during testing (Fig. 3D). According to this model, over the course of testing, 52\% (26/50) of Category TI participants did not appreciably improve (i.e. had distributions of estimated slopes overlapping with zero), whereas only $22 \%(13 / 60)$ of Standard TI 

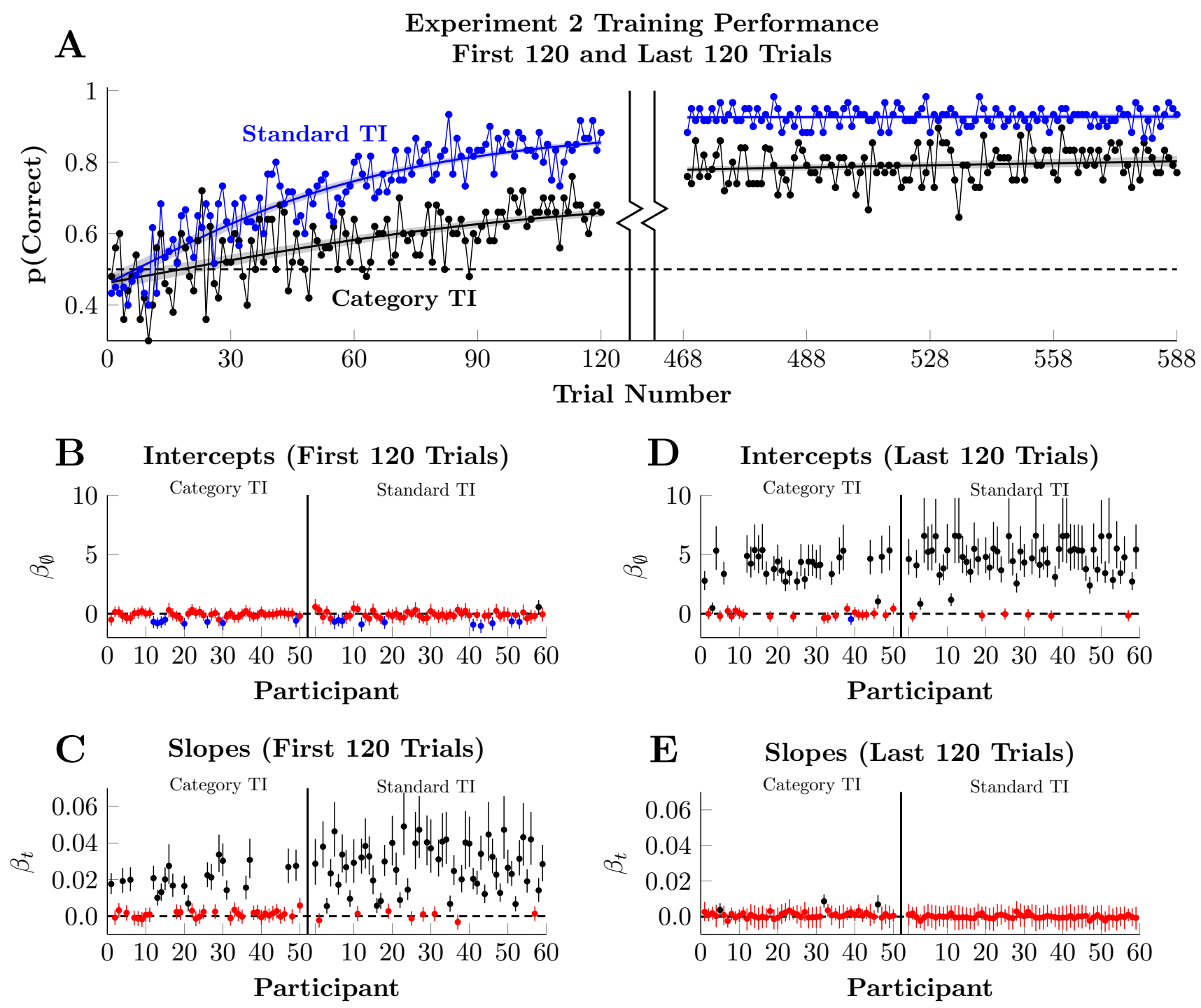

Figure 2. Logistic regression model of TI performance during training trials. A. Mean response accuracy for the Category TI condition (black) and the Standard TI condition (blue) during the first and last 120 trials of training. Points represent observed means, whereas curves represent the estimates mean and $95 \%$ credible interval based on a multi-level model. B. Intercept parameters ( $\beta_{\emptyset}$ in Equation 1) for each participant, based on a multi-level model of performance during the first 120 trials. Whiskers on each estimate denote its $95 \%$ posterior credible interval. Estimates in red include zero within their credible intervals, whereas estimates in black consistently exceed zero and estimates in blue consistently fall below zero. C. Slope parameters ( $\beta_{t}$ in Equation 1) for each participant during the first 120 trials. D. Intercept parameters for each participant during the last 120 trials. E. Slope parameters for each participant during the last 120 trials.

participants did not appreciably improve during training. However, it is important to keep in mind that the participant-level estimates are based on only 60 trials of data (i.e. ten presentations apiece of each of the critical pairs), and in almost all cases, there was insufficient power to detect an effect of symbolic distance, or a distance-by-trial interaction.

Interpreting the distance effect at the start of testing (Fig. 3C) and its interaction with the learning rate (Fig. 3E) are difficult to interpret, as participant-level results were underpowered with respect to the number of free parameters in the model. Overall, a hypothetical "average participant" displayed a distance effect, but with only one testing session each, the participant-level estimates remained highly 

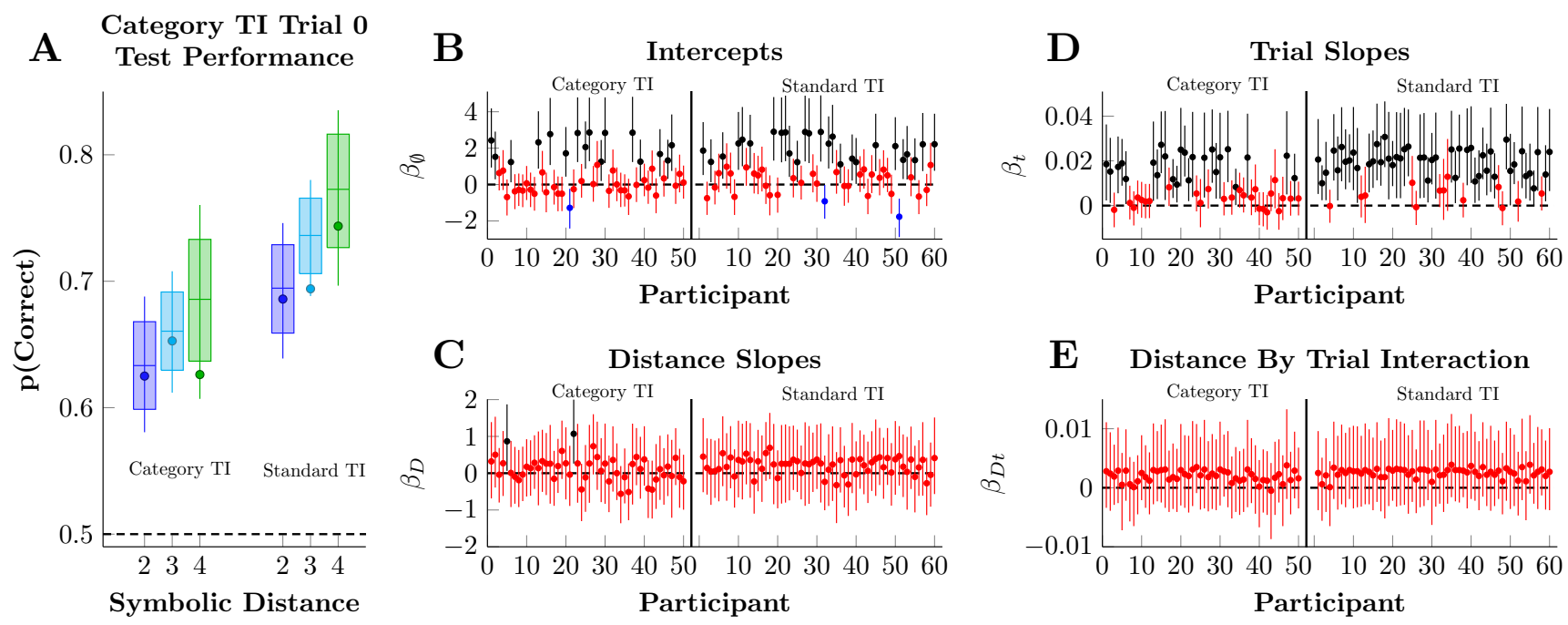

Figure 3. Logistic regression model of TI performance of only critical pairs $(\mathrm{BD}, \mathrm{CE}, \mathrm{DF}, \mathrm{BE}, \mathrm{CF}$, and $\mathrm{BF})$ during testing trials. A. Estimated critical pair response accuracy for the Category TI and Standard TI conditions on the first trial of testing. Points represent observed means, whereas boxes represent the $80 \%$ credible interval and whiskers represent the $95 \%$ credible interval, based on a multi-level model. B. Intercept parameters ( $\beta_{\emptyset}$ in Equation 2$)$ for each participant. Whiskers on each estimate denote its $95 \%$ posterior credible interval. Estimates in red include zero within their credible intervals, whereas estimates in black consistently exceed zero and estimates in blue consistently fall below zero. C. Slope parameters with respect to symbolic distance ( $\beta_{D}$ in Equation 2$)$ for each participant, measuring the symbolic distance effect at the start of testing. $\mathbf{D}$. Slope parameters with respect to trial number ( $\beta_{t}$ in Equation 2$)$ for each participant, measuring the overall learning rate. $\mathbf{E}$. Distance-by-trial interaction parameters $\left(\beta_{D t}\right.$ in Equation 2$)$ for each participant, measuring the change in the symbolic distance effect over time.

uncertain. These were suggestive, however. Whereas a clear majority of participants in the Standard TI condition $(80 \%, 48 / 60)$ had parameter distributions whose mean was greater than zero, only $60 \%$ (25/50) of Category TI participants did so. Furthermore, the effect of partial pooling in the model likely shrank any underlying difference between conditions. This analysis suggests that although participants on average showed evidence of transitive inference in both conditions, participants in the Category TI condition performed less well overall. To rigorously confirm this rest and test for a distance effect required a follow-up analysis, which we describe below.

Our first approach to address the low power on display in Figure 3 was to perform simple logistic regressions (using Equation 1, with only slope and intercept terms) in order to assess performance for each of the 21 pairs at the beginning of testing. These estimates of response accuracy are presented in Figure 4, which provides an assessment of the critical pairs that is independent of the model assumptions made by Equation 2. Although performance overall appeared less accurate in the Category TI condition than in the standard TI condition overall, the most striking difference is in the six critical pairs (at symbolic distances 2,3 , and 4), showing signs of a distance effect in the Standard TI condition but appearing comparatively flat in the Category TI condition. This provides an additional indication that although performance in the Category TI condition was above chance, it may nevertheless be qualitatively different than performance in the Standard TI condition.

In order to characterize the differences between conditions, we further examined the learning process itself for individual participants. Learning curves like those plotted in Figure 2 are often misleading, because they can take learning processes that are abrupt and, as a result of averaging, give the impression that they change gradually (Gallistel et al., 2004). Although multi-level models provide a means of obtaining participant-level estimates, logistic regression carries the implicit assumption of gradual learning. 


\section{Category TI}

A Trial 0 Pairwise Test Performance

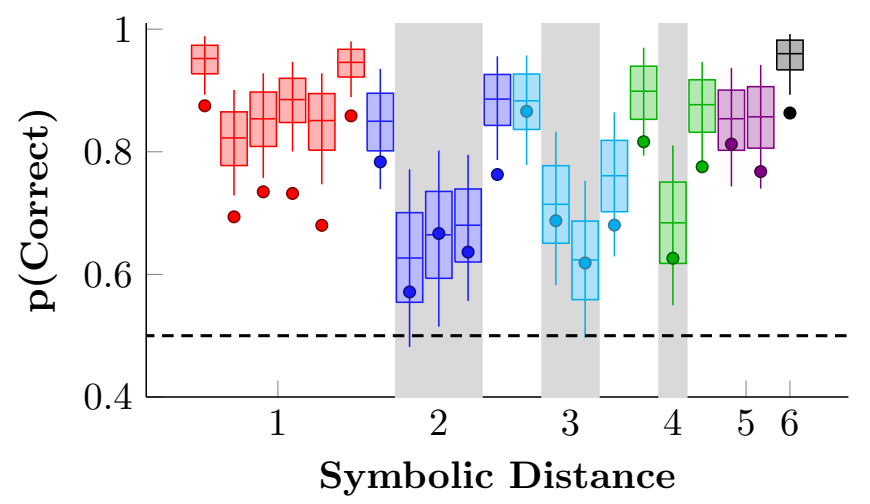

B Standard TI

B Trial 0 Pairwise Test Performance

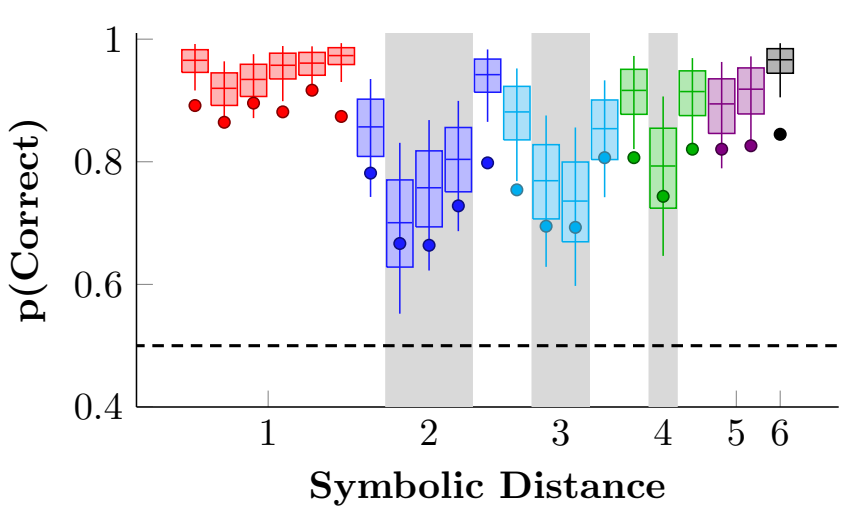

Figure 4. Logistic regression model of TI performance of each pair at the start of testing, evaluated independently of one another using Equation 1 and sorted by symbolic distance. Points represent observed means, whereas boxes represent the 80\% credible interval and whiskers represent the $95 \%$ credible interval, based on a multi-level model. Critical pairs are shaded with a gray backdrop. A. Estimated response accuracy for the Category TI pairs. B. Estimated response accuracy for the Standard TI pairs.

To determine what accounted for the differences in learning between groups observed in Figures 2 and 3 , a subject-level analysis was performed. For each trial of training, or of testing, the cumulative number of correct responses by trial $\mathrm{t}$ was calculated, denoted by $C_{t}$. The likelihood function describing the probability of a correct response is given by the beta distribution. Given a flat prior distribution, the posterior density for a participant's "probability of a correct response" is given by beta $\left(\frac{C_{t}+1}{t+2} \mid C_{t}+1, t-C_{t}+1\right)$. This can be used to specify an upper and lower "credible range" for a participant's accuracy. We designated any participant whose $95 \%$ credible range excluded chance during training as a "learner" and those participants whose posterior distribution overlapped with 0.5 as "non-learners."

Figure 5 shows how the cumulative probability of correct responses evolved during training trials (top) and testing trials (bottom) for participants in the Category TI condition (left) and Standard TI condition (right). The line plotting a participant's evolution was colored red until it crossed the threshold that excluded chance performance from its $95 \%$ credible interval for the last time, after which it was colored blue. The blue circles below each plot reflect the trial at which that threshold was crossed, while the blue diamonds to the right of each plot reflect the overall accuracy achieved by each participant. The box-and-whisker diagram accompanying each reports the mean and bootstrapped confidence intervals of only the learners.

In this analysis of the training phase (Figure 5 top), learning was clearly slower in general for the Category TI condition (an average of 178 trials to exceed chance), than for the Standard TI condition (an average of 89 trials to exceed chance). Participants were also less likely to be learners in the Category TI condition: $38 \%$ (19/50) of Category TI participants were non-learners during training, compared to $10 \%(6 / 10)$ of Standard TI participants. However, this difference can potentially be attributed to the added difficulty of needing to recognize the categories in use. Although the Category TI learners took longer to do so, the fairly smooth upward trajectories of their cumulative response accuracies reflect fairly high response accuracy during the last few trials of training.

A more dramatic difference is observed in the analysis of the testing phase (Figure 5 bottom). Learners of both groups exceeded the threshold of chance responding fairly quickly (within 50 trials for most), but whereas most Standard TI participants made very few errors and completed testing with accuracies 


\section{A}
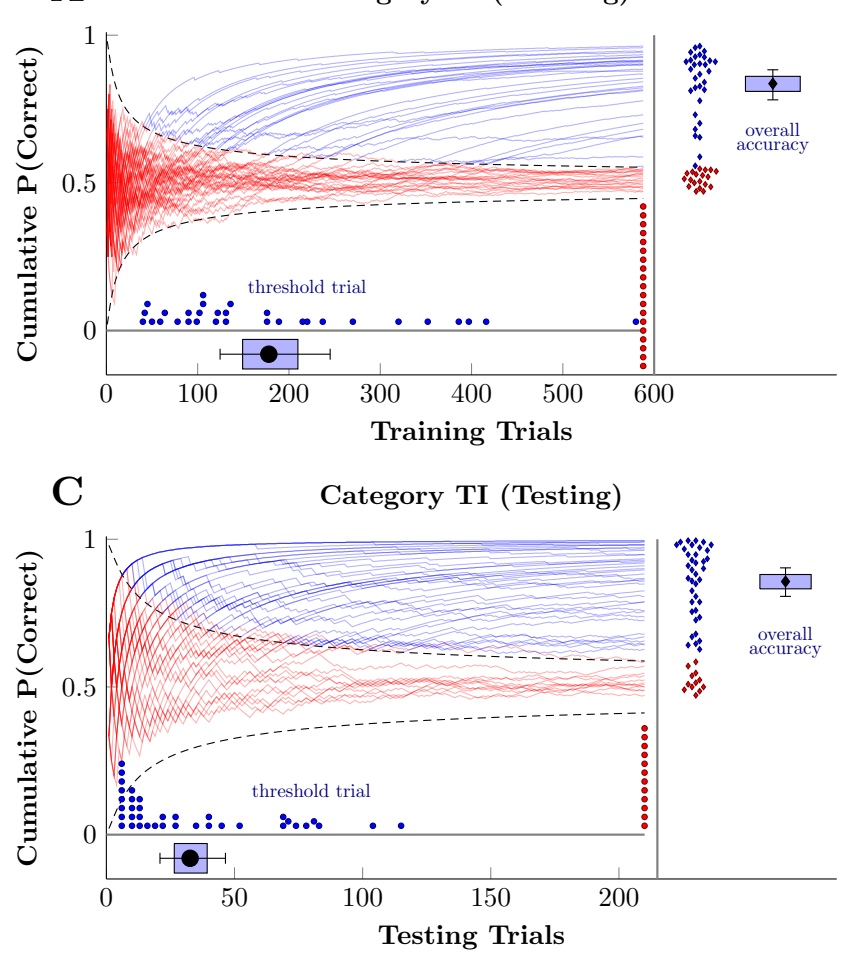

B Standard TI (Training)

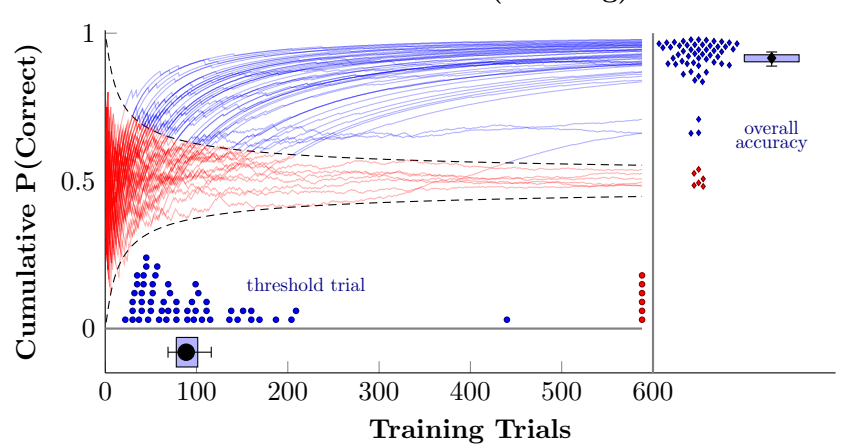

D

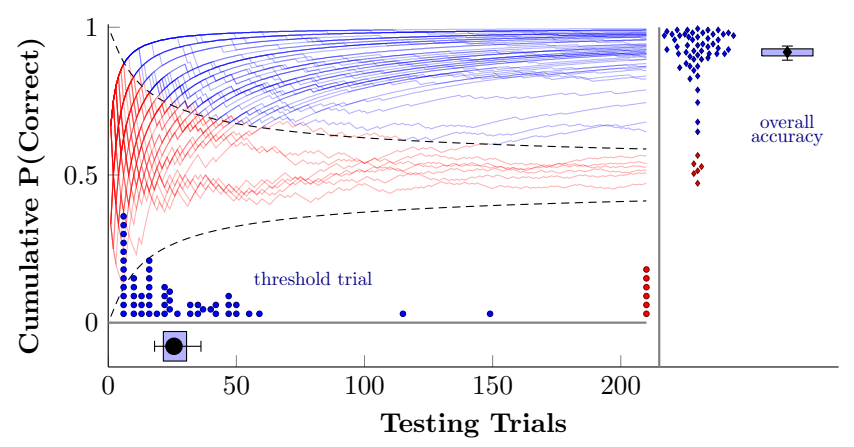

Figure 5. Cumulative proportion of correct responses for each participant. When this proportion for the last time crosses the threshold of excluding chance from its 95\% credible interval, the line is plotted in blue, at a position marked below the plot with a blue circle; otherwise, the line is plotted in red. The final overall proportion of correct responses in the phase is marked to the right of the plot with a diamond. Participants who cross the threshold are designated "learners." The blue box-and-whisker plots show the mean threshold trial and overall accuracy for learners only, with $80 \%$ (box) and 95\% (whisker) bootstrapped uncertainties. A. Cumulative response accuracy during training for Category TI participants. B. Cumulative response accuracy during training for Standard TI participants. C. Cumulative response accuracy during testing for Category TI participants. D. Cumulative response accuracy during testing for Standard TI participants.

above $80 \%$, learners in the Category TI condition showed a wide range of response accuracies, with a very uniform spread of response accuracies between chance and near-perfect performance. Thus, most participants during Category TI testing, even those who did well during training, had difficulty learning the categories of all 21 pairs of categorical items during testing.

One potential implication of Figure 5 is that because many more participants had not yet displayed evidence of having learned the task during the training phase, average performance at the start of testing (as characterized by Figures 3 and 4) would have consisted of different proportions of learners and nonlearners. To address this, we performed a new logistic regression analysis of performance on the six critical pairs that omitted any participants who were non-learners by the end of the training phase. In order to ensure that the Category TI and Standard TI estimates were independent, we examined participants in each condition separately. Finally, to address the possibility that our analysis in Figure 3 was underpowered, we omitted the variable of "trials" and limited out analysis to only include responses during either the first block or last block of trials in the testing phase (each also assessed independently). This allowed us to examine whether a symbolic distance effect was evident among the learners.

Figure 6 presents the results of these four analyses. On the one hand, participants responded above chance to the critical pairs in both the Category TI and Standard TI conditions at both the beginning and end of testing, as shown by the estimates mean performance (Figures 6A and 6D). This effect was also 


\section{A Critical Pairs Test Performance}
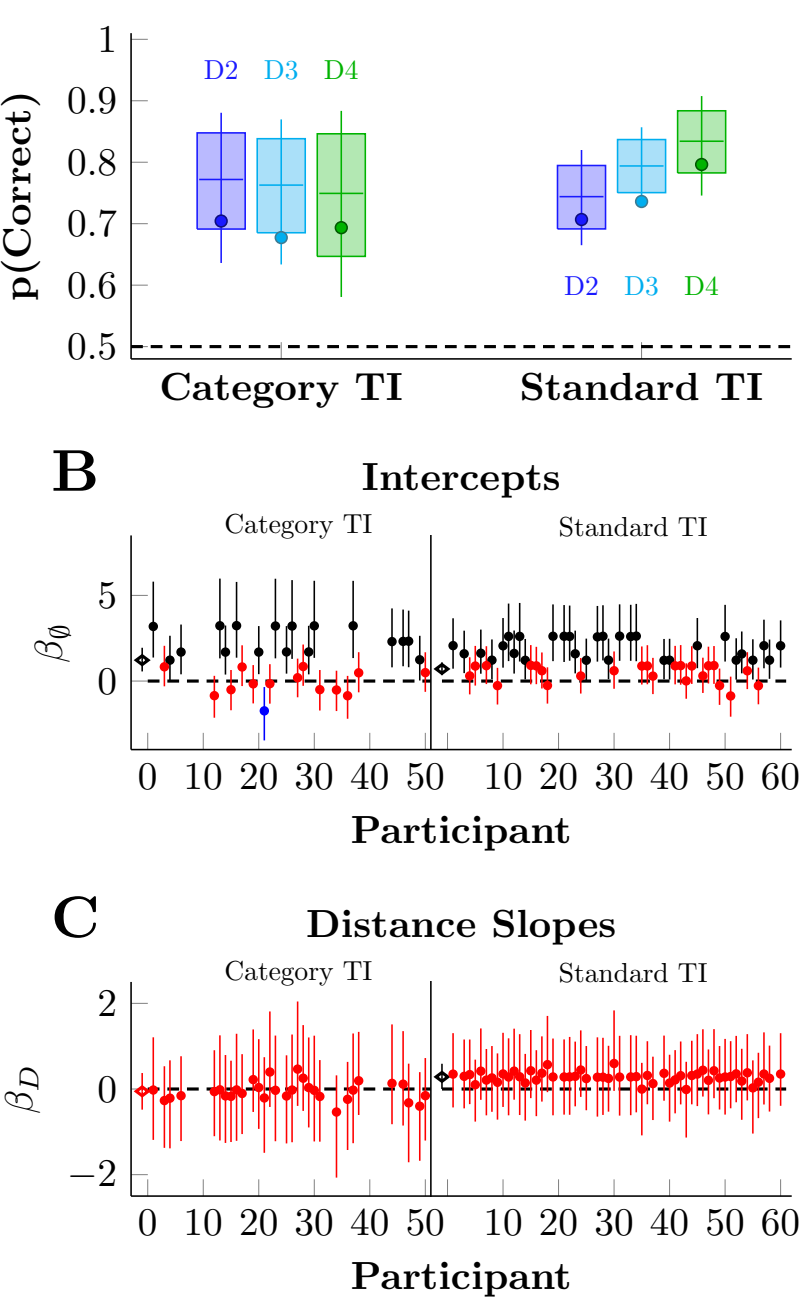

D Critical Pairs Test Performance

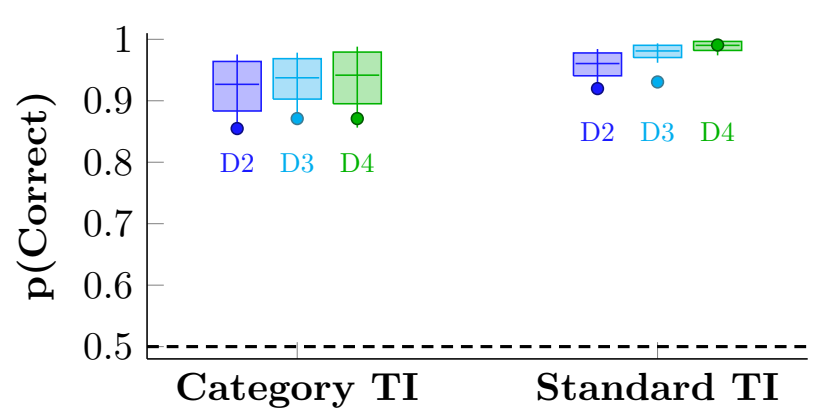

E Intercepts

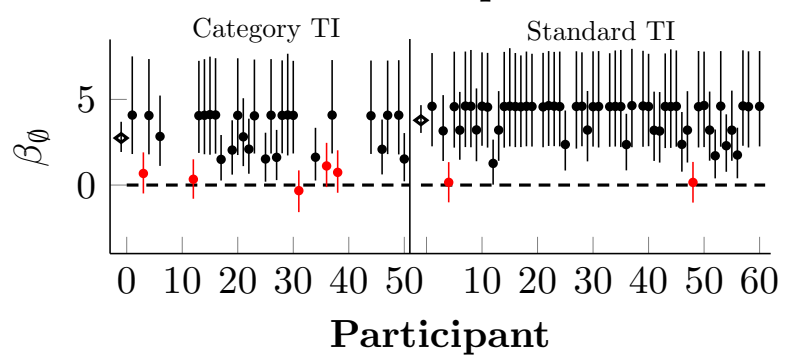

F Distance Slopes

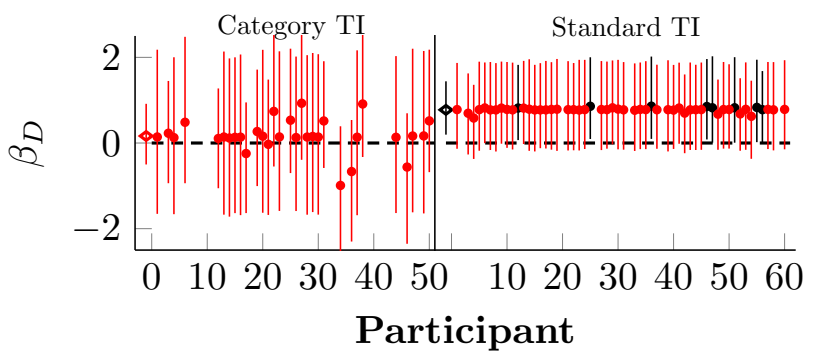

Figure 6. Logistic regression model of TI performance of only critical pairs (BD, CE, DF, BE, CF, and BF) during the first and last response block of the test phase. These analyses included only those participants who were designated "learners" in Figure 5. A. Estimated critical pair response accuracy for the Category TI and Standard TI conditions during the first block of testing. Points represent observed means, whereas boxes represent the $80 \%$ credible interval and whiskers represent the $95 \%$ credible interval, based on a multi-level model. B. Intercept parameters for each learner during the first block of testing. Whiskers on each estimate denote its $95 \%$ posterior credible interval. Estimates in red include zero within their credible intervals, whereas estimates in black consistently exceed zero and estimates in blue consistently fall below zero. C. Slope parameters with respect to symbolic distance for each learner during the first block of testing, measuring the symbolic distance effect. D. Estimated critical pair response accuracy for the Category TI and Standard TI conditions during the last block of testing. E. Intercept parameters for each learner during the last block of testing. F. Slope parameters with respect to symbolic distance for each learner during the last block of testing, measuring the symbolic distance effect.

demonstrated, as a population average, by the logistic regression's intercept terms (Figures 6B and 6E). However, when examining the distance slope term in the regression, although the Standard TI participants did on average show a symbolic distance effect, Category TI participants did not, either during the first block of testing (Figure 6C) or the last block of testing (Figure 6F). Thus, although many participants who had above-chance performance during training also exceeded chance on critical pairs at the start of testing, and despite nearly all participants exceeding chance by the end of testing, only participants in the Standard 
TI condition displayed a distance effect in their response accuracy at any point during the testing phase.

\section{DISCUSSION}

Participants performed one of two transitive inference (TI) tasks, in which they learned the implicit order of lists of seven different elements. Lists were first trained using adjacent pairs of list elements. They were then tested using all possible pairs. In the Standard TI task, seven randomly selected stimuli were drawn from 7 familiar categories ("cats," "flowers," etc.). These same stimuli were used throughout the experiment, although each participant was assigned a different set of 7 such images, in a different order. In the Category TI task, the stimuli in the ordered list were drawn from the same 7 categories, but novel exemplars were used on each trial. Although participants in this condition never saw any exemplar more than once, most learned the order of the categories represented by these exemplars. In both task conditions, participants were trained on pairs of adjacent stimuli and then tested using all possible pairs. Our data therefore show that participants can perform transitive inferences, as determined by above-chance performance on critical pairs at the start of testing, with respect to entire categories of stimuli, and not merely with respect to specific stimuli.

Transitive inference about categories has been studied previously in non-human subjects. Tanner et al. (2015) trained rhesus macaques on a 5-item Category TI task with a similar trial structure to ours, albeit with a much longer training phase. Even though the monkeys had no prior experience with tasks of categorization, their performance displayed all the hallmarks of successful transitive inference, including above-chance performance on the critical pair BD and a symbolic distance effect (SDE). The SDE is widely considered as the strongest evidence that participants use an internal representation of list order when performing serial learning tasks, including TI.

SDEs are generally evident in studies of transitive inference. Monkeys were able to demonstrate a SDE during learning of 5-item lists using TI, and using the simultaneous chaining paradigm (D'Amato and Colombo, 1990; Jensen et al., 2013). SDEs emerge as an automatic consequence of TI when learning lists that are specified as a one-dimensional spatial continuum and on which feedback is represented by a fuzzy distribution (Jensen et al., 2019). These results suggest that if humans treat the Category TI similarly to monkeys, then human performance in the Category TI condition should resemble that seen in the Standard TI condition.

Reports of SDEs in human studies of TI are somewhat less common, but this is largely the result of human studies often not testing all possible pairs, or using lists that are not long enough to allow comparison of critical pairs of varying length. However, such effects usually emerge when a sufficiently rich dataset is collected. For example, (Acuna et al., 2002) reported a distance effect in both response accuracy (Fig. 6) and reaction times (Fig. 7). Given that humans have typically displayed symbolic distance effects in standard TI paradigms and that monkeys showed and SDE for category TI, we expected to see the SDE for both the Standard TI and Category TI conditions in this study.

However, even though there was evidence of transitive inference in participant performance on both the Category TI and the Standard TI conditions, only the latter yielded evidence of SDEs. Furthermore, participants were less likely to achieve above-chance responding overall during learning (i.e. becoming "learners") in the Category TI condition, and those who responded above chance generally performed less well than Standard TI participants at testing. That was true even though the participants of both groups received the same minimal verbal instructions, and completed the same number of trials. It therefore appears that different cognitive processes gave rise to knowledge of transference inference during Category TI learning versus Standard TI learning. 


\section{Categorization \& Serial Learning}

Our procedure for training categorization overcomes certain limitations of the more widely used approach for assessing binary categorization (Jensen and Altschul, 2015). When training is limited to two categories are used throughout training, the correct response can often be determined by a default strategy. Hence, participants may develop expertise at visual discrimination, but not more abstract aspects of categorization. For example, when learning to discriminate the categories "faces" and "houses", participants can respond correctly solely on the basis of "number of sharp angles." Training with a variety of categories subverts categorization on the basis of a simple dichotomy and is therefore a more rigorous test of category learning. The present study adds to an existing literature showing that category learning can occur in parallel with serial learning (Altschul et al., 2017).

Tasks of rule-based category learning imply that category structures can be learned with the implementations of explicit signals, such as feedback (Ashby and Vucovich, 2016). However, as exemplars of categories increase, there may be a continuum of inferred learning from this exemplar memorization to a more dynamic learning of prototype abstraction (Ell et al., 2017). In our experiment, the exemplars for each category were consistently, and randomly different. Therefore, there is no obvious rule-based strategy for determining the categories or their ranking. In this case, task difficulty increases with the number of exemplars in each category, and may account for the observation that category TI learning was slower, and there were more non-learners, compared to standard TI.

\section{Transitive Inference without a Symbolic Distance Effect}

Certain systematic differences between human and non-human TI performance complicate simple generalization to humans of models validated in non-human subjects. Such comparisons must be made cautiously, as the mechanisms and strategies that support TI in humans may differ from those seen in non-humans (Moses et al., 2006). For example, human participants generally display high levels of accuracy during testing with adjacent training pairs, potentially from some combination of familiarity and specific memorization of those pairs. Consequently "distance 1" pairs generally have high accuracy relative to other pairs during testing in most human studies of TI, a result that is evident in our data (Figure 4). Additionally, both human participants, and non-human subjects, also tend to display large terminal item effects (TIE), characterized by elevated response accuracy on pairs that include either the first or last list item (reviewed by Jensen, 2017). The dual effects of training set familiarity and TIE have led some researchers to suggest that the SDE should not be the "signature" of TI performance because SDEs are routinely not seen in human TI performance (Wright and Howells, 2008). We should note, however, that the experiments described by Wright and Howells trained participants on lists containing only 4 items, meaning that any SDEs would have been confounded by TIEs.

When humans are trained on longer lists that include multiple critical pairs of varying symbolic distance, an SDE that is independent of those two confounding effects can be assessed. In our experiment, participants trained on a Standard TI task do display SDEs when responding to those pairs (Figure 6C). As noted above Acuna et al. (2002) have also reported that SDEs are observed for critical pairs when TI is trained using lists containing at least 6 items, thus presenting at least three critical pairs (see also Frank et al., 2005; Libben and Titone, 2008; Moses et al., 2010; Jensen et al., 2015). In the case of performance of human participants on lists containing 6 or more items, there should be a strong expectation of an SDE in response to critical pairs. What's puzzling is the absence of an SDE in participants trained on the Category TI task. The absence of a SDE is not consistent with any published computational models of TI of which we are aware, whether associative or cognitive.

One possibility is that reasoning about categories made participants in some way less aware of the relationships between list positions. Several studies (Frank et al., 2005; Smith and Squire, 2005; Libben 
and Titone, 2008) have reported substantial differences in performance in participants who are unable to describe the task requirements at the end of a session of TI ("non-aware"), from those who can give an approximate description of what the task requires ("aware"). However, this does not appear to be a sufficient account of our results for three reasons. First, our participants were reliably above chance on all critical pairs, whereas the non-aware participants in the afore-mentioned studies generally score at chance levels on at least some critical pairs. Secondly, all three of the above studies performed experiments with 6-item lists and found that the critical pairs BD and CE (symbolic distance 2) had lower average accuracy than the critical pairs BE (symbolic distance 3) in both the aware and unaware conditions. Thus, while awareness of the task demands might plausibly be lower in the Category TI task, "unaware" participants should still be expected to display SDEs.

Both the relatively high accuracy of performance in our Category TI participants, and the absence of an SDE, suggest that our results were not due to a lack of awareness. The role of awareness can also be questioned by verbal descriptions by our participants of the task that we obtained during debriefing, which occurred at the end of each experimental session. Most participants used phrases like "order," "hierarchy," "category," and "X beats $Y$ " in response to an open query at the beginning of the debriefing phase to describe the experiment, before the experimenter revealed anything about the nature of the task requirements.

\section{Levels of Transitive Inference}

Marr (1982) famously distinguished between the computational level of understanding an information processing system ("what" the system does) and the algorithmic level ("how" the system does it). The debate about transitive inference is hampered by the being broadly defined as a phenomenon at the computational level: If an organism exceeds chance on a critical pair, then scholars will generally agree that "transitive inference" has occurred. Given the same evidence, some favor an associative model (Vasconcelos, 2008); others, a cognitive model (Jensen, 2017). Attempts to decipher the algorithmic level have depended on pushing the boundary of evidence beyond merely exceeding chance on critical pairs, such as testing whether models correctly predict effects like SDEs and TIEs. The lack of an SDE in Category TI (despite its clear appearance after a corresponding amount of Standard TI training) may reflect some essential difference between two different algorithms for transitive inference in human brains. It may also be a consequence of the algorithms used by primate brains generally to categorize stimuli.

For example, lateral intra-parietal neurons (LIP) have been implicated serial learning using TI paradigms (Munoz et al., 2020). Given that LIP is also implicated in explicitly spatial forms of cognition (Bisley and Goldberg, 2003; Chafee et al., 2007), the hypothesis that the "spatial reasoning" hypothesis of TI used to explain SDEs might be supported by circuitry suited to spatial reasoning appears at least plausible. The apparent connection between graded parietal activation and SDEs (Hinton et al., 2010) is also consistent with that view. However, LIP has also been implicated in categorization (Swaminathan and Freedman, 2012), using circuitry that appears to be functionally independent of spatial encoding (Rishel et al., 2013). Thus, the computational phenomenon of TI might emerge from processing taking place in both spatial and categorical parietal cortex, but those two regions might implement different algorithms. This unnerving possibility suggests that no single theory of TI will satisfactorily explain the many ways in which TI is performed. Instead, the study of TI in the brain may follow a trajectory similar to that of

reward prediction errors: A general behavior of neural circuits throughout the brain, rather than a single form of learning localized to a specific region. 


\section{Future Directions}

There is rarely a single clear confounding task variable on which a null result can be blamed. For example, perhaps an SDE would have been observed in the Category TI condition if participants had more training. While this would not change how surprising our current results are, given the amount of training in our experiment, it may turn out an SDE would emerge given sufficient training. On the other hand, in our experience, the SDE emerges early in all-pairs testing. In one experiment with four nonhuman primates, an SDE emerged in standard TI with as few as 6 training trials (Jensen et al., unpublished observations presented at SFN 2019).

Alternatively, our result might be explained by excessive familiarity, not with the Category TI task, but with the categories themselves. Participants may have had extensive experience distinguishing cats from birds. If indeed Category TI relies on particular computations, they may be relatively inflexible because of their extensive experience making non-hierarchical judgments about these categories. Another question is whether Category TI can be used to train entirely novel categories (e.g. stimuli generated on the basis of abstract mathematical rules). Learning a category from scratch in a serial learning context may be sufficient for those categories to display a more typical pattern of distance effects.

Our study also makes clear the importance of including a control group when studying novel variations on existing tasks. Had the Category TI task been run in isolation, it would have been tempting to characterize the absence of an SDE as an "inconsequential null," - one that could be attributed to insufficient statistical power. Without the opportunity to compare performance of participants trained on a Standard TI task, it would not have been possible to assess the performance of the Category TI group. If we are to explain TI at anything deeper than a descriptive level, it is vital that we report our results on a trial-by-trial basis that controls for task variables, such as amount of training, to make performance comparisons as precise as possible.

\section{Funding}

This study was supported by NIH-MH081153 and NIH-MH111703 (VPF and HST).

\section{Author Contributions}

The study was designed by GJ, TK, VPF, and HST. Data were collected by TK, CM, and SSB. Data were analyzed by GJ. The paper was written by GJ, TK, VPF, and HST, with input from CM and SSB.

\section{REFERENCES}

Acuna, B. D., Eliassen, J. C., Donoghue, J. P., and Sanes, J. N. (2002). Frontal and parietal lobe activation during transitive inference in humans. Cerebral Cortex, 12:1312-1321.

Altschul, D., Jensen, G., and Terrace, H. S. (2017). Perceptual category learning of photographic and painterly stimuli in rhesus macaques (macaca mulatta) and humans. PLOS ONE, 12:e0185576.

Ashby, F. G. and Vucovich, L. E. (2016). The role of feedback contingency in perceptual category learning. Journal of Experimental Psychology: Learning, Memory, \& Cognition, 42:1731-1746.

Bisley, J. W. and Goldberg, M. E. (2003). Neuronal activity in the lateral intraparietal area and spatial attention. Science, 299:81-86.

Bogale, B. A., Aoyama, M., and Sugita, S. (2011). Categorical learning between 'male' and 'female' photographic human faces in jungle crows (corvus macrorhynchos). Behavioural Processes, 86:109118.

Bryant, P. E. and Trabasso, T. (1971). Transitive inference and memory in young children. Nature, 232:456-458. 
Carpenter, B., Gelman, A., Hoffman, M., Lee, D., Goodrich, B., Betancourt, M., Brubaker, M. A., Guo, J., Li, P., and Riddell, A. (2017). Stan: A probabilistic programming language. Journal of Statistical Software, 76:1-32.

Chafee, M. V., Averbeck, B. B., and Crowe, D. A. (2007). Representing spatial relationships in posterior parietal cortex: single neurons code object-referenced position. Cerebral Cortex, 17:2914-2932.

Croft, W. and Cruse, D. A. (2004). Cognitive Linguistics. Cambridge University Press, Cambridge, UK.

Cromer, J. A., Roy, J. E., and Miller, E. K. (2010). Representation of multiple, independent categories in the primate prefrontal cortex. Neuron, 66:796-807.

Cuaya, L. V., Hernandez-Perez, R., and Concha, L. (2016). Our faces in the dog's brain: Functional imaging reveals temporal cortex activation during perception of human faces. PLOS ONE, 11:e0149431.

D'Amato, M. R. and Colombo, M. (1990). The symbolic distance effect in monkeys (cebus apella). Animal Learning \& Behavior, 18:133-140.

David, H. (1992). Transitive inference in rats (rattus norgevicus). Journal of Comparative Psychology, 106:342-349.

Ell, S. W., Smith, D. B., Peralta, G., and Hélie, S. (2017). The impact of category structure and training methodology on learning and generalizing within-category representations. Attention, Perception, \& Psychophysics, 79:1777-1794.

Ferrera, V. P., Yanike, M., and Cassanello, C. (2009). Frontal eye field neurons signal changes in decision criteria. Nature Neuroscience, 12:1458-1462.

Frank, M. J., Rudy, J. W., Levy, W. B., and O'Reilly, R. C. (2005). When logic fails: Implicit transitive inference in humans. Memory \& Cognition, 33:742-750.

Freedman, D. J. and Assad, J. A. (2016). Neuronal mechanisms of visual categorization: An abstract view on decision making. Annual Review of Neuroscience, 33:129-147.

Freedman, D. J., Riesenhuber, M., Poggio, T., and Miller, E. K. (2001). Categorical representation of visual stimuli in the primate prefrontal cortex. Science, 291:312-316.

Freedman, D. J., Riesenhuber, M., Poggio, T., and Miller, E. K. (2003). A comparison of primate prefrontal and inferior temporal cortices during visual categorization. Journal of Neuroscience, 23:5235-5246.

Gallistel, C. R., Fairhurst, S., and Balsam, P. D. (2004). The learning curve: Implications of a quantitative analysis. Proceedings of the National Academy of Sciences USA, 101:13124-13131.

Grosenick, L., Clement, T. S., and Fernald, R. D. (2007). Fish can infer social rank by observation alone. Nature, 445:429-432.

Hinton, E. C., Dymond, S., von Hecker, U., and Evans, C. J. (2010). Neural correlates of relational reasoning and the symbolic distance effect: involvement of parietal cortex. Neuroscience, 168:138-148.

Jensen, G. (2017). Serial learning. In Call, J., Burghardt, G. M., Pepperberg, I. M., Snowdon, C. T., and Zentall, T., editors, APA Handbook of Comparative Psychology, pages 385-409. American Psychological Association, Washington, DC.

Jensen, G. and Altschul, D. (2015). Two perils of binary categorization: why the study of concepts can't afford true/false testing. Frontiers In Psychology, 6:Article 168.

Jensen, G., Altschul, D., Danly, E., and Terrace, H. S. (2013). Transfer of a serial representation between two distinct tasks by rhesus macaques. PLOS ONE, 8:e70285.

Jensen, G., Munoz, F., Alkan, Y., Ferrera, V. P., and Terrace, H. S. (2015). Implicit value updating explains transitive inference performance: The betasort model. PLOS Computational Biology, 11:e1004523.

Jensen, G., Terrace, H. S., and Ferrera, V. P. (2019). Discovering implied serial order through model-free and model-based learning. Frontiers In Neuroscience, 13:Article 878.

Kanwisher, N., McDermott, J., and Chun, M. M. (1997). The fusiform face area: a module in human extrastriate cortex specialized for face perception. Journal of Neuroscience, 17:4302-4311. 
Kendrick, K. M., da Costa, A. P., Leigh, E., Hinton, M. R., and Pierce, J. W. (2001). Sheep don't forget a face. Nature, 414:165-166.

Lazareva, O. F., Freiburger, K. L., and Wasserman, E. A. (2004). Pigeons concurrently categorize photographs at both basic and superordinate levels. Psychonomic Bulletin \& Review, 11:1111-1117.

Lazareva, O. F. and Wasserman, E. A. (2006). Effect of stimulus orderability and reinforcement history on transitive responding in pigeons. Behavioural Processes, 72:161-172.

Lewandowsky, S. (2011). Working memory capacity and categorization: Individual differences and modeling. Journal of Experimental Psychology: Learning, Memory, and Cognition, 37:720-738.

Libben, M. and Titone, D. (2008). The role of awareness and working memory in human transitive inference. Behavioural Processes, 77:43-54.

Marr, D. C. (1982). Vision: A Computational Investigation in the Human Representation and Processing of Visual Information. W. H. Freeman and Company, New York, NY, USA.

McGonigle, B. O. and Chalmers, M. (1977). Are monkeys logical? Science, 267:694-696.

McGonigle, B. O. and Chalmers, M. (1992). Monkeys are rational! Quarterly Journal of Experimental Psychology Section B: Comparative and Physiological Psychology, 45:189-228.

Moses, S. N., Brown, T. M., Ryan, J. D., and McIntosh, A. R. (2010). Neural system interactions underlying human transitive inference. Hippocampus, 20:894-901.

Moses, S. N., Villate, C., and Ryan, J. D. (2006). An investigation of learning strategy supporting transitive inference performance in humans compared to other species. Neuropsychologia, 44:1370-1387.

Munoz, F., Jensen, G., Kennedy, B. C., Terrace, H. S., and Ferrera, V. P. (2020). Learned representation of implied serial order in posterior parietal cortex. Scientific Reports, pages DOI: 10.1038/s41598-02065838-9.

Neuringer, A. and Jensen, G. (2010). Operant variability and voluntary action. Psychological Review, 117:972-993.

Piaget, J. (1921). Une forme verbal de la comparison chez l'enfant. Archive de Psychologie, 18:141-172.

Rishel, C. A., Huang, G., and Freedman, D. J. (2013). Independent category and spatial encoding in parietal cortex. Neuron, 77:969-979.

Silverman, J. L., Gastrell, P. T., Karras, M. N., Solomon, M., and Crawley, J. N. (2015). Cognitive abilities on transitive inference using a novel touchscreen technology for mice. Cerebral Cortex, 25:1133-1142.

Smith, C. and Squire, L. R. (2005). Declarative memory, awareness, and transitive inference. Journal of Neuroscience, 25:10138-10146.

Sonnweber, R., Ravignani, A., and Fitch, W. T. (2015). Non-adjacent visual dependency learning in chimpanzees. Animal Cognition, 18:733-745.

Spierings, M. J. and ten Cate, C. (2016). Budgerigars and zebra finches differ in how they generalize in an artificial grammar learning experiment. Proceedings of the National Academy of Science USA, 113:E3977-E3984.

Swaminathan, S. K. and Freedman, D. J. (2012). Preferential encoding of visual categories in parietal cortex compared with prefrontal cortex. Nature Neuroscience, 15:315-320.

Tanner, N., Jensen, G., Ferrera, V. P., and Terrace, H. S. (2015). Inferential learning of serial order of perceptual categories by rhesus monkeys (Macaca mulatta). Journal of Neuroscience, 37:6268-6276.

Terrace, H. S. (2011). Missing links in the evolution of language. In Dehaene, S. and Christen, Y., editors, Characterizing Consciousness: From Cognition to the Clinic?, pages 1-25. Springer-Verlag, Berlin, Germany.

Tibbetts, E. A., Agudelo, J., Pandit, S., and Roijas, J. (2019). Transitive inference in polistes paper wasps. Biology Letters, 15:20190015.

Tsao, D. Y., Freiwald, W. A., Knutsen, T. A., Mandeville, J. B., and Tootell, R. B. H. (2003). Faces and 
objects in macaque cerebral cortex. Nature Neuroscience, 6:989-995.

Van der Jeugd, A., Goddyn, H., Laeremans, A., Arckens, L., D’Hooge, R., and Verguts, T. (2009). Hippocampal involvement in the acquisition of relational associations, but not in the expression of a transitive inference task in mice. Behavioral Neuroscience, 123:109-112.

Vasconcelos, M. (2008). Transitive inference in non-human animals: An empirical and theoretical analysis. Behavioural Processes, 78:313-334.

Wright, B. C. and Howells, D. (2008). Getting one step closer to deduction: Introducing an alternative paradigm for transitive inference. Thinking \& Reasoning, 14:244-280.

Wright, H. F., Wilkinson, A., Croxton, R., Graham, D. K., Harding, R. C., Hodkinson, H. L., Keep, B., Cracknell, N. R., and Zulch, H. E. (2017). Animals can assign novel odours to a known category. Scientific Reports, 7:Article 9019. 